\title{
Respond, don't react: The influence of mindfulness training on performance monitoring in older adults
}

\author{
Colette M. Smart ${ }^{1,2}$ • Sidney J. Segalowitz ${ }^{3,4}$
}

Published online: 2 October 2017

(C) Psychonomic Society, Inc. 2017

\begin{abstract}
A sizeable body of literature demonstrates positive effects of mindfulness training on brain, behavior, and psychological processes in both novice and expert practitioners as compared to non-meditators. However, only more recently has research begun to examine the specific mechanisms by which mindfulness exerts these effects. In the current study, we used event-related potentials (error-related negativity (ERN), error positivity (Pe)) to test the hypothesis that performance monitoring is one such mechanism. We conducted a randomized controlled trial in healthy older adults $(n=36)$, relevant because markers of performance monitoring are known to decline with normal aging. Compared to an active control condition, mindfulness participants showed an increase in the ERN, without an increase in the Pe. Participants in both groups reported a reduction in selfreport of anxiety and self-judgment of one's own mental functioning, indicating the subjective impression of benefit from each intervention type. The current results are important insofar as they support the purported self-regulatory functions of mindfulness (i.e., learning to respond, not react), as well as
\end{abstract}

Colette M. Smart

csmart@uvic.ca

1 Department of Psychology, University of Victoria, PO Box 1700 STN CSC, Victoria, BC V8W 2Y2, Canada

2 Institute on Aging and Lifelong Health, University of Victoria, Victoria, BC V8W 2Y2, Canada

3 Department of Psychology, Brock University, L25 3A1, St. Catharines, ON, Canada

4 Jack and Nora Walker Centre for Lifespan Development Research, Brock University, L25 3A1, St. Catharines, ON, Canada demonstrating that such positive effects can be obtained in an older adult sample, both of which have important implications for intervention.

Keywords Mindfulness · Performance monitoring · Error-related negativity $\cdot$ Older adults $\cdot$ Subjective cognitive decline

\section{Introduction}

Over the last two decades, a robust literature has developed regarding the impact of mindfulness training on cognitive and emotional functioning. Disseminated through programs such as mindfulness-based stress reduction (Kabat-Zinn, 1990) and mindfulness-based cognitive therapy (Segal, Williams, \& Teasdale, 2012), evidence has been found for enhancements in self-reported psychological functioning in meditation-naïve medical (Bohlmeijer et al., 2010) and psychiatric populations (Chiesa, Serretti, \& Jakobsen, 2013; Hoffman, Sawyer, Witt, $\&$ Oh, 2010). Research also suggests enhanced neural and behavioral functioning for healthy individuals, both novice and expert practitioners (Chiesa, Calati, \& Serretti, 2011; Lutz, Slagter, Dunne, \& Davidson, 2008; MacLean et al., 2010) as well as neurologic populations (Azulay et al., 2013; Chen et al., 2011; Levine et al., 2011; Novakovic-Agopian et al., 2010; Zylowska et al., 2008). Much of the early literature focused on establishing group differences between those who do and do not practice mindfulness. Research is now beginning to turn to a more nuanced understanding of the actual mechanisms whereby mindfulness exerts its effects. The current study sought to understand changes in performance monitoring as one such mechanism for change. 


\section{Performance monitoring as a mechanism for mindfulness}

In terms of how mindfulness might achieve its effects, a core premise of the training is learning to "respond, not react" (Kabat-Zinn, 1990). That is, one learns to monitor one's mental state (i.e., self-regulation of attention), ascertain deviations from one's present moment experience, and gently bring the mind back without judgment, criticism, or impulsive action (i.e., responding with neutral observation or loving-kindness). Mindfulness is not a finite end-point but rather a moment-tomoment process, hence the purported role for self-regulation in mindfulness (Bishop et al., 2004).

In the cognitive electrophysiology literature, the term performance monitoring has been used to describe the process of monitoring, detection, and evaluation of behavioral and environmental consequences in order to facilitate the most adaptive behavioral responses given the current situation (van Noort \& Segalowitz, 2012). Evidence suggests that neural control of these processes is largely associated with prefrontal cortex - particularly medial prefrontal cortex (mPFC) areas such as anterior cingulate cortex (ACC) (Holroyd \& Yeung, 2012) - governing both cognitive control of motor behavior (Polli et al., 2005; Ridderinkhof et al., 2004,b), as well as affective and motivational responses to feedback (Diekhof et al., 2011; Etkin et al., 2011; Ridderinkhof et al., 2004; Schnider et al., 2005). This integration of cognitive and emotional processing seems particularly relevant to the discussion of mechanisms of mindfulness.

Two event-related potential components are particularly associated with performance monitoring - the error-related negativity (ERN) and the error positivity (Pe). The ERN is a response-locked component that occurs in association with errors in a speeded task, occurring shortly before the commission of the error and peaking usually within $100 \mathrm{~ms}$ subsequent to the response. While there are divergent theories on its functional significance, most agree that the ERN is elicited by modulation of attention in response to errors (Friedman, 2012; Gehring, Liu, Orr, \& Carp, 2012; van Noordt, Campopiano \& Segalowitz, 2016; van Noordt, Desjardins, \& Segalowitz, 2015; van Noordt \& Segalowitz, 2012), whether elicited by affective or cognitive factors. Increased ERN responses have also been associated with increased negative affect, including state and trait anxiety (Aarts \& Pourtois, 2015; Hajcak, 2012; Olvet \& Hajcak, 2008). The Pe peaks between $200 \mathrm{~ms}$ and $400 \mathrm{~ms}$ after an error, also linked to mPFC (van Veen \& Carter, 2002) but with a more posterior, centroparietal topography (Gehring et al., 2012). Possibly the least well understood of the error monitoring components from a functional perspective, the Pe has been linked to both conscious awareness of errors and affective/evaluative response to errors (Overbeek, Nieuwenhuis, \& Ridderinkhof, 2005). The Pe has also been proposed as a P3 component (specifically, $\mathrm{P} 3 \mathrm{~b}$ ) occurring in response to errors with affective salience
(Davies, Segalowitz, Dywan \& Pailing, 2001; Overbeek et al., 2005).

Examining the impact of mindfulness training on these components could illuminate its mechanisms of selfregulation or performance monitoring. For example, does mindfulness increase attentiveness to errors (i.e., increased ERN) but decrease affective salience of those errors (i.e., decreased Pe)? Compared to the attention literature, few studies directly examine the impact of mindfulness on performance monitoring, and the available results are mixed.

Larson, Steffen, and Primosch (2013) recruited meditationnaïve participants and randomized half of them to a very brief (i.e., $\sim 15 \mathrm{~min}$ ) mindfulness intervention. Compared to the control group, there was no intervention effect on either behavioral performance on the Eriksen flanker task or the latency or amplitude of the associated ERN, but they did find that the amplitude of the Pe was significantly reduced following mindfulness training, suggesting either less conscious reaction to or less affective salience of errors. At the other end of the meditative continuum, Teper and Inzlicht (2013) found that long-term meditators of different backgrounds had greater ERN amplitudes compared to non-meditators, suggesting that perhaps longer-term practice is needed to see robust differences in the ERN. In fact, years of meditation experience and meditation frequency significantly predicted ERN amplitude, even after controlling for demographic variables as well as general accuracy on the Stroop task. Emotional acceptance was positively correlated with ERN amplitude, and emotional acceptance (and, to a lesser degree, ERN amplitude) mediated the relationship between meditation experience and performance on the Stroop task. The findings on emotional acceptance are particularly interesting, given that some studies show a positive association between ERN amplitude and negative affect (Aarts \& Pourtois, 2015; Hajcak, 2012; Olvet \& Hajcak, 2008). Thus, mindfulness is not simply about increasing the ERN, but also balancing that with greater non-reactivity and non-judgment.

Schoenberg et al. (2014) examined the impact of 12 weeks of mindfulness-based cognitive therapy in persons with attention-deficit/hyperactivity disorder. In contrast to Teper and Inzlicht (2013), results revealed an overall attenuation in ERN amplitude following mindfulness training as compared to an increase in the ERN in the waitlist control group. This dissociation between studies in terms of the ERN effect is likely to be related to the population under study (i.e., healthy "experts" vs. clinical participants) and illustrates the selfregulatory aspect of mindfulness. They also found a nonsignificant trend toward a reduction in errors on a continuous performance task as well as a significant increase in Pe amplitude at frontocentral sites following mindfulness training. For an attention-deficit/hyperactivity disorder population, increasing awareness and salience of errors has adaptive value, again illustrating the nuanced impact of mindfulness depending on the context and population under study. 
In an innovative design, Saunders et al. (2016) sought to use the ERN and the Pe to understand mechanisms of mindfulness that may dissociate between responses to cognitive versus affective stimuli. Healthy volunteers were randomized to a brief training in mindful attention focused on either emotions or thoughts. Each training group received an approximate 9-min focused attention audio instruction. For the remainder of the 13-min instruction, the emotion-focused group was asked to focus on their emotions and inquire to themselves about what they were feeling right now, and to simply be aware of those feelings. The thought-focused group was conversely asked to be aware of the thoughts going through their mind. All participants completed a baseline go/no-go task, followed by the 13-min mindful induction, and then a final go/no-go task, during which the ERN and Pe were recorded. Results indicated a greater ERN response in the emotion-focused group, whereas focusing on thoughts did not impact the ERN. Moreover, no effect was found for either group on the Pe. While the functional significance of the ERN has been debated, particularly with respect to cognitive versus affective stimuli, the authors interpreted the results to indicate that mindfulness may exert its effects on performance monitoring primarily through attention to affective stimuli. While this design is innovative in trying to dissociate mechanisms of mindfulness as a function of stimulus type, one possible limitation of the study is the ecological validity with regards to typical mindfulness training programs such as mindfulnessbased stress reduction. That is, in the course of such programs, participants are not instructed to only focus on one type of stimulus (i.e., cognitive vs. affective). Moreover, more often that not, participants frequently observe that self-referential thinking in and of itself generates negative affect (Farb et al., 2007) and by attending to and letting go of such thoughts, negative emotional responses attenuate. Thus, it may be interesting in the future to ascertain whether participants in longerterm meditation training (e.g., 8 weeks) perform similarly as to the participants in Saunders et al.'s (2016) experiment.

Finally, very recent work by Bing-Canar, Pizzuto, and Compton (2016) examined the impact of mindfulness of breathing on error-related alpha suppression (ERAS), the phenomenon whereby oscillatory EEG activity in the alpha frequency band is suppressed following errors as opposed to correct responses (Carp \& Compton, 2009; van Noordt et al., 2017). That is, occurrence of an error signals the individual to deploy greater attentional resources to the task at hand, which leads to a concomitant decrease in alpha-band activity. In this study, the authors sought to build on the aforementioned studies by Larson et al. (2013) and Teper and Inzlicht (2013) by determining whether a brief (i.e., $15 \mathrm{~min}$ ) mindfulness of breathing intervention could increase ERAS in response to the Stroop task as compared to an audio-listening condition. Participants were healthy undergraduates who ranged in prior exposure to mindfulness meditation practice on a scale of 1 (no exposure) to 10 (prior practice) (mean score $=5.6, \mathrm{SD}=0.4$ ). Although not the main focus of the study, the ERN, Pe, and theta activity were taken as secondary measures. The authors confirmed their hypothesis of an overall ERAS effect, present in both conditions but more pronounced in the mindfulness group. However, they found no significant effects of experimental condition on either the ERN or the Pe. Larson et al. (2013), who also used a 15-min intervention in primarily meditation-naïve participants, similarly found a lack of effects on the ERN, but they did find a reduction in the Pe amplitude. The discordance in findings between these two studies suggests that $15 \mathrm{~min}$ may be an insufficient dose to provide a reliable effect on performance-monitoring components, and that lengthier training may be needed to produce more robust findings.

\section{Aims and hypotheses for the current study}

In the current study, we combined both performance monitoring components (i.e., ERN, Pe) to understand the impact of mindfulness on self-regulation in healthy older adults, something which, to our knowledge, has not been done before. The available developmental literature indicates age-related declines in the ERN (Friedman, 2012; Nieuwenhuis et al., 2002; Pietschmann, Simon, Endrass, \& Kathmann, 2008; Wild-Wall, Willemssen, \& Falkenstein, 2009), suggesting that the impact of mindfulness on this event-related potential component might be more evident in this population. Additionally, the regulation of cognition and emotion become competing demands in older adulthood (Peters, Hess, Västfjäll, \& Auman, 2007), which might allow for further exaggeration of differences between the event-related potential components for older adults undergoing mindfulness training.

Primary hypotheses Based on the limited available literature and theoretical understanding, we hypothesized that mindfulness training would increase the ERN (i.e., attentiveness to errors) while decreasing the Pe (i.e., affective salience of errors), and that this would be associated with a decrease in negative affect and difficulties with mood regulation as well as increased self-reported mindfulness. We expected pre/post changes in the error components specifically following mindfulness training and not our active control condition. This is due to the fact that only the mindfulness condition contained active cognitive training, particularly with regards to attention control, whereas the control condition did not. Furthermore, recent reviews suggest that cognitive/behavioral effects of mindfulness are most evident in long-term practitioners (Chiesa et al., 2011); thus, we did not anticipate significant behavioral effects above and beyond repeated exposure to the tasks pre-/post-intervention. 
Secondary hypotheses A subset of our participants was classified as having subjective cognitive decline. This recently identified syndrome pertains to older adults who complain or shown concern about perceived decline in cognitive function, despite performing within normal limits on standardized clinical assessment and maintaining independent activities of daily living (Jessen et al., 2014). Longitudinal studies estimate that as many as $60 \%$ of older adults with subjective cognitive decline will decline to mild cognitive impairment and Alzheimer's dementia over an approximately 15 -year period (Reisberg et al., 2008). Thus, we had two secondary hypotheses: (1) compared to healthy older adults, the subjective cognitive decline group would show differences in our measures of performance monitoring, and (2) following mindfulness training, persons with subjective cognitive decline would show a stronger effect of self-regulation compared to healthy controls.

\section{Methods}

\section{Participants}

Healthy older adults aged 65-80 years were prospectively recruited through radio and print media to participate in a study that had two aims: (1) to understand cognitive, behavioral, and self-report measures that discriminate individuals with subjective cognitive decline from healthy controls; and (2) to ascertain whether targeted intervention could improve the cognitive and emotional functioning of individuals with subjective cognitive decline. The current report represents one specific question of interest extracted from the second aim. Our screening and classification methods have been documented in detail elsewhere (Smart et al., 2014). In brief, we used a two-part screening process that involved a combination of standardized neuropsychological testing and self and informant-report questionnaires to rule out individuals with amnestic $\mathrm{MCI}$ and dementia. We classified remaining individuals as either having subjective cognitive decline or as being a healthy control based on their response to the following question: "Are you concerned or worried that you are experiencing significant decline in your thinking abilities, more than just normal aging?" We excluded individuals with a positive neurologic history (e.g., traumatic brain injury, stroke, neurodegenerative disease), as well as those who could not communicate in English, had no reliable informant, could not undergo structural MRI scanning (for a separate analysis), and could not participate through the entirety of the intervention. 114 individuals underwent an initial phone screen in phase 1, with 65 individuals undergoing further in-person screening in phase 2. Forty-two individuals were eligible to enroll in the intervention, and 38 were randomized to intervention.

\section{Measures}

Self-report measures Anxiety was measured using the total score on the Adult Manifest Anxiety Scale, Elderly Version (Lowe \& Reynolds, 2006). Difficulties in mood regulation were measured using the Negative Mood Regulation Scale (Catanzaro \& Mearns, 1990). Mindfulness was measured using the Five Facet Mindfulness Questionnaire (Baer et al., 2008); for our primary focus on performance monitoring, we specifically examined the Non-Judge and Non-React subscales.

Experimental tasks As noted above, this study represents one piece of a larger study examining baseline differences in cognitive function associated with subjective cognitive decline, as well as cognitive, emotional, and neural response to mindfulness training. As part of baseline testing, participants underwent a battery of standardized neuropsychological and experimental-cognitive tasks, including four event-related potential tasks that were counterbalanced within the task administration. These included a go/no-go task with associated P3 ERP, results of which we have reported on previously elsewhere (Smart, Segalowitz, Mulligan, Koudys, \& Gawryluk, 2016). The ERN and Pe were obtained in conjunction with a form of the Eriksen flanker task (Eriksen \& Eriksen, 1974) used in previous studies (Santesso \& Segalowitz, 2009). Four computer-generated visual displays of five letters were presented (S's and H's), displayed $250 \mathrm{~ms}$ each with an ISI of $1,000 \mathrm{~ms}$. The target letter ( $\mathrm{S}$ or $\mathrm{H}$ ) was flanked by either congruent (SSSSS, HHHHH) or incongruent (SSHSS, HHSHH) letters (120 trials of each type, totalling 480 test trials), and the target letter required a key-press. Behavioral indices included reaction time and accuracy. Stimuli were presented on a standard computer monitor using E-Prime version 2 (Psychology Software Tools, 2012). ${ }^{1}$

\section{Procedures}

The Human Research Ethics Boards of the University of Victoria and Vancouver Island Health Authority approved this study. Participants completed two levels of screening to determine eligibility before being enrolled into the final study (see Smart et al., 2014, for further details of the screening

\footnotetext{
${ }^{1}$ We also included a task that elicits a feedback-related negativity (FRN) to an unexpected unfortunate event, indicating failure on that trial of the task ending in a loss of points in a video-type game (see Segalowitz et al., 2012, for details). The results somewhat mimicked those of the ERN but weakly: The interaction of Time $\times$ Intervention was significant $(F(1,30)=4.96, p=.034$, partial eta-squared $=.142$ ), whereby the FRN increased following mindfulness training and marginally decreased after psychoeducation. Unfortunately, neither of these single changes were statistically reliable, $t=1.69, p=.11$ and $t=$ $1.64, p=.12$, respectively; the intervention groups did differ at the end, $t=$ $2.56, p=.015$, but not at the beginning, $t=.26, p=.80$. Thus, there is the suggestion that the mindfulness intervention may demonstrate an increase in the FRN response in this paradigm if the statistical power were greater, but our current results are not powerful enough to demonstrate this.
} 
procedures). Participants worked for no more than $150 \mathrm{~min}$ in any one testing block and were provided with breaks within each time-block as requested, to minimize the effects of fatigue on performance. During the electrophysiology session participants completed two additional tasks not related to the primary performance monitoring hypothesis of this report.

Self-report questionnaires Administration of self-report questionnaires occurred in a quiet testing room with several participants simultaneously. Measures were scored according to the test creator's guidelines, producing either mean or total scores for further analysis.

Cognitive electrophysiology EEG was recorded at a sampling rate of $500 \mathrm{~Hz}$ with an online analog bandpass of 0.016-100 Hz and amplified with a 32-channel BrainAmp system (Brain Products). Using Abralyt 2000 electrode gel and $\mathrm{Ag} / \mathrm{AgCl}$ electrodes mounted on an electrode cap, signals were recorded according to the extended 10- to 20-system of electrode placement from 28 scalp sites Fp1/2, F7/8, F3/4, Fz, FC5/6, FC1/2, FCz, C3/4, Cz, CP5/6, CP1/2, P7/8, P3/4, Pz, $\mathrm{PO} 7 / 8, \mathrm{O} 1 / 2$ and from left mastoid (M1) all referred to the right mastoid (M2), and AFz as ground. Sensors on the outer canthus of each eye and inferior to the left eye were used to monitor horizontal and vertical eye movements/blinks, respectively. All electrode impedances were maintained below $10 \mathrm{k} \Omega$ throughout the recording session.

EEG data were processed through independent component analysis (ICA) in order to isolate non-cortical sources as latent components, including EOG, muscle movements and heartbeat artifacts (as outlined in Desjardins \& Segalowitz, 2013 and van Noordt et al., 2015). After removing these artifacts, the remaining independent components were projected back to the scalp. (Unfortunately, with only 28 scalp sites it was not possible to derive independent cortical components.) For the stimulus-locked ERP scoring, trial segments were then divided into segments $200 \mathrm{~ms}$ before stimulus onset to $1,000 \mathrm{~ms}$ afterwards, and baseline corrected to the 200-ms period before stimulus onset. For the response-locked ERN and Pe, the trials were segmented into epochs starting $800 \mathrm{~ms}$ before the response to $1,000 \mathrm{~ms}$ after the response. The baseline correction was to the period $600-400 \mathrm{~ms}$ before the response. This was done so that the baseline would capture a period of time before the stimulus onset and avoid the positivity that precedes the ERN component.

Intervention procedures We employed a single-blind, randomized controlled trial design, whereby each diagnostic group (healthy control vs. subjective cognitive decline) was split in half and randomly assigned to participate in either mindfulness training tailored specifically for older adults or psychoeducation on cognitive aging (each $\mathrm{n}=19$ ). Participants from the healthy control and subjective cognitive decline groups were intermixed within the intervention groups and were blind to this classification to reduce stereotype threat and poor performance due to expectations related to diagnostic assignment (Kit, Tuokko, \& Mateer, 2008). Group leaders were similarly blind to the diagnostic group of participants.

Mindfulness training was delivered using an 8-week manualized protocol entitled Wisdom Mind. This was based on Kabat-Zinn's (1990) mindfulness-based stress reduction but specifically tailored to older adults, informed by knowledge of cognitive aging and principles of cognitive rehabilitation (Smart, 2012). Standard practices were introduced, including the body scan, mindfulness of breathing, and gentle yoga. Approximately the first half of the course was spent learning techniques to enhance focused attention mindfulness, while the second half of the course was spent learning techniques focused on open monitoring mindfulness (Lutz et al., 2008). Particularly relevant to the current analysis, in the second half of the course, participants were also introduced to two practices related to emotion regulation: the first, "emotional weather," related to the ability to observe one's current emotional state without judgment or commentary. The second, "loving-kindness," is often taught in mindfulness-based stress reduction, but in this program it was contextualized in terms of normalizing cognitive slips in older adults and decreasing emotional reactivity when such slips occurred. Participants were also introduced to the concept of cognitive reserve (Stern, 2009) and how mindfulness could be used to enhance openness to trying new activities, that could in turn support cognitive reserve and act as a buffer against age-related cognitive decline. Daily home practice (i.e., body scan, mindfulness of breathing, gentle yoga, loving-kindness) was encouraged and facilitated by specially recorded audio CDs and a workbook, also used by the group leader to administer the program. The workbook included a homework log, in which participants were encouraged to record daily practice.

The control condition, psychoeducation on cognitive aging, was delivered via a manualized protocol that has been empirically validated for use in healthy older adults with cognitive concerns (Troyer, 2001; Wiegand et al., 2013). This 5-week group included information on normal and non-normal age-related cognitive changes as well as factors that can influence memory such as stress, mood/anxiety, medications, and certain medical conditions. Participants were given strategies to boost memory performance and opportunities to practice these within group sessions, as well as between sessions at home.

Baseline testing occurred during an approximate 4-week period prior to starting the intervention. The mindfulness training group began their intervention at week one, followed by the psychoeducation group 3 weeks later. The intervention groups were completed at the same time (i.e., week eight) and all participants underwent post-intervention testing within 2 weeks of completion. While a 3-week difference between 
intervention conditions might appear to be a confound, the psychoeducation condition did not involve active cognitive training, thus adding three further weeks of intervention would not be expected to produce any further improvement in cognitive ability. Moreover, each condition was delivered according to a standardized protocol previously determined to provide the required "dose" of each intervention.

\section{Results}

As noted above, 38 individuals were enrolled in intervention groups; demographic information for these participants is presented in Table 1. The stringent screening criteria employed at the time of recruitment led to a reduced sample size. However, this was not out of the norm for comparable clinical studies on mindfulness training in neurologic samples (Azulay et al., 2013; Chen et al., 2011; Levine et al., 2011; Zylowska et al., 2008), and also led to a more a homogeneous sample of participants. Further strengths of the study included the use of a randomized controlled trial design and an active control group (unlike the wait-list control groups used in many comparable studies).

There were no significant group differences at baseline in age $(t(36)=.344, p=.733)$, ethnicity $\left(\chi^{2}(1)=1.973, p=\right.$ $.160)$, marital status $\left(\chi^{2}(4)=5.087, p=.279\right)$, number of years of education $(t(36)=.561, p=.733)$, or estimated premorbid function $(t(36)=-.705, p=.485)$. The only significant difference was in gender $\left(\chi^{2}(1)=4.26, p>0.04\right)$; there were more men relative to women in the healthy control group, with the opposite pattern observed in the subjective cognitive decline group. In terms of attrition, one participant failed to complete the intervention due to health reasons, and a second completed the intervention but failed to complete post-testing also due to health reasons, representing one participant from each of the healthy control and subjective cognitive decline groups, respectively. In addition, four participants were dropped due to extremely messy EEG data in the flanker task. This resulted in complete pre/post-test data on our key measures in the flanker task for 32 participants (healthy control $=19$, with ten and nine in the mindfulness training and psychoeducation interventions, respectively; subjective cognitive decline $=13$, with six and seven in the two intervention groups, respectively).

\section{Neural and behavioral measures of performance monitoring}

The Eriksen flanker task was used to generate the averaged ERPs of error trials yielding the ERN and Pe, as well as reaction time and accuracy. As is often the case, the correct trials did not yield a clearly scorable negativity in the ERN time period (i.e., CRNs) consistently enough to provide a comparison with the error trials and therefore we calculated the ERN effects where the correct trial waveforms were subtracted from the error trial waveforms. The ERN effect was calculated at $\mathrm{Fz}, \mathrm{FCz}$ and $\mathrm{Cz}$ as the difference between the maximum negativity in the -50 to $+200 \mathrm{~ms}$ surrounding the response and the positivity that precedes it. All three sites yielded highly reliable ERN effects: Fz: $F(1,13)=56.3, p<.001$, partial eta squared $=.813 ; \mathrm{FCz}: F(1,27)=127.2, p<.001$, partial eta squared $=.825 ; \mathrm{Cz}: F(1,28)=180.3, p<.001$, partial eta squared $=.866$. However, our analyses focus on the results from FCz for several reasons. First, the ERN is normally maximal here and this is the site that is normally used (Gehring et al., 2012) and indeed this is what we have found (e.g., Santesso \& Segalowitz, 2009). In fact, in the current data, the ERN at $\mathrm{Fz}$ is considerably attenuated compared to that at $\mathrm{FCz}$ and the variation across participants within cells is considerably larger at both $\mathrm{Fz}$ and $\mathrm{Cz}$ compared with $\mathrm{FCz}$. Second, we have also found that when a high-density montage

Table 1 Characteristics of participants enrolled into the intervention, based on self-identification as subjective cognitive decline (SCD) or healthy controls $(\mathrm{HC})(\mathrm{n}=38)$

\begin{tabular}{lll}
\hline & $\begin{array}{l}\mathrm{HC}(\mathrm{n}=23) \\
(\mathrm{M}, \mathrm{SD})\end{array}$ & $\begin{array}{l}\mathrm{SCD}(\mathrm{n}=15) \\
(\mathrm{M}, \mathrm{SD})\end{array}$ \\
\hline Age (years) & $70.0(3.45)$ & $69.60(3.58)$ \\
Sex $(\mathrm{M} / \mathrm{F})^{*}$ & $14 / 9$ & $4 / 11$ \\
Ethnicity & $100 \%$ Caucasian & $91.7 \%$ Caucasian \\
Marital status & & $60.0 \%$ \\
$\quad$ Partnered (domestic or marital) & $69.6 \%$ & $40.0 \%$ \\
$\quad$ Single/separated/divorced/widowed & $30.4 \%$ & $16.40(2.69)$ \\
Education (years) & $17.09(4.11)$ & $113.20(13.00)$ \\
WAIS-IV Test of premorbid function** & $110.48(10.66)$ & $10 / 5$ \\
Self-report of prior psychiatric illness $(\mathrm{Y} / \mathrm{N})$ & $18 / 5$ & \\
\hline
\end{tabular}

*Significant at $p<.05$

**Wechsler Test of Adult Intelligence, Fourth Edition (Wechsler, 2008) 
is used with ICA to derive a single latent component sourced to the medial frontal cortex that captures the ERN variance at the scalp, it is at FCz that it is maximal (Van Noordt et al., 2015) and this is the cleanest measure of ERN at the scalp. Third, we found that many of our participants did not yield scorable data at the Fz site, reducing considerably the data available for analysis when we try to include site as a factor in the statistical designs.

To test both for baseline (pre-intervention) differences between diagnostic groups as well as for intervention effects, we conducted a Time (Time 1/Time 2) $\times$ Group (healthy control vs. subjective cognitive decline) $\times$ Intervention (mindfulness vs. psychoeducation) mixed ANOVA for data at FCz. Contrary to our hypothesis, there was no effect of Group $(F$ $<1)$ but Time was significant $(F(1,27)=14.6, p=.001$, partial eta squared $=.351$ ), with ERN amplitude increasing from $3.827 \mu \mathrm{V}$ to $5.380 \mu \mathrm{V}$. Intervention as a main effect was not significant, which is not surprising since assignment to interventions was random at Time $1(F(1,27)=1.25, p=, 274$, partial eta squared $=.044)$. Similarly, the interaction of Group $\times$ Intervention was not significant $(F(1,27)=3.36, p$ $=.078$, pes $=.111)$. Of more interest was the significant interaction of Time $\times$ Intervention $(F(1,27)=5.52, p=.026$, partial eta squared $=.170$ ), whereby the ERN increased amplitude with mindfulness training more than with psychoeducation, as shown in Figs. 1 and 2. Follow-up tests indicated that the psychoeducation group did not show an effect of Time $(t(1,15)=1.54, p=.144)$, while the mindfulness intervention $\operatorname{did}(F(1,14)=3.10, p=.008)$. This suggests that the interaction is due specifically to the mindfulness intervention increasing the within-subject ERN.

The number of trials contributed to each person's ERP varied as a function of the number of errors they committed adjusted

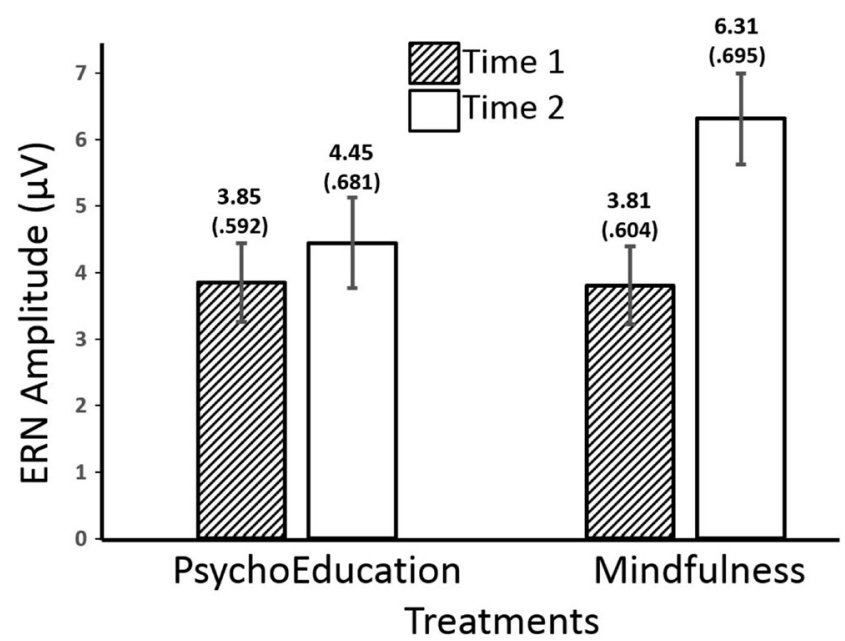

Fig. 1 Collapsing across diagnostic group (Healthy Controls vs. Subjective Cognitive Decline), the error-related negativity (ERN) at $\mathrm{FCz}$ increased significantly more following mindfulness training than psychoeducation. Figures above the bars are mean values with standard errors in parentheses; the latter are also shown by vertical lines by trials eliminated due to artifacts. The averages (and standard deviations) were 25.9 (11.4) and 22.8 (12.4) for the pre- and post-intervention sessions, respectively. The individual differences, perhaps reflecting the degree of impulsiveness, were highly reliable with $r=.716, p<.001$. Of particular interest, however, is whether the number of trials contributed to effects, as one might expect that those committing more errors would have an attenuated ERN. To examine this, we ran ANOVAs again with the number of trials contributed from Time 1 or from Time 2 entered as a covariate. In both cases the primary effect remained $(F(1,26)=5.734, p=.024$, partial eta squared $=.181$ and $F(1,26)=5.324, p=.029$, partial eta squared $=.170$, for Time 1 and Time 2 number of trials covaried respectively).

The Pe was scored with the same task at site Pz, and because of how it is scored could yield values for correct as well as error trials. As expected, a reliably larger $\mathrm{Pe}$ amplitude at $\mathrm{Pz}$ (averaged over 200 to $600 \mathrm{~ms}$ post-response) was associated with more trials on which errors $(.61 \mu \mathrm{V})$ occurred than when correct $(.02 \mu \mathrm{V})$ responses were given $(F(1,28)=14.9, p=$ .001 , partial eta-squared $=.348$ ), although the absolute magnitude was small. Again, contrary to predictions, the Pe was not sensitive to diagnostic group or interactions associated with group (all $F \mathrm{~s}<1$ ). However, there was a significant Time $\times$ Intervention interaction regarding the overall positivity of the $\operatorname{Pe}(\mathrm{F}(1,28)=5.88, p=.022$, partial eta-squared $=.174)$, whereby mindfulness training produced a slight reduction in the Pe $(.27$ to $.15 \mu \mathrm{V})$ while psychoeducation increased the Pe (.26 to $.59 \mu \mathrm{V}$ ) (see Fig. 3). Perhaps because of the small sample size, the 3-way interaction of Correct vs. Error trials $\times$ Time $\times$ Intervention did not reach significance, $F(1,28)=2.41$, $p=.132$, partial eta-squared $=.079$. However, as one might expect, the Time $\times$ Intervention interaction was clearly present on error trials, $F(1,28)=4.70, p=.039$, partial eta-squared $=$ .144 , and not on correct trials, $F<1$. Follow-up post hoc tests indicated that intervention groups did not differ on Pe for correct trials at Time $1(t<1)$ or Time $2(t(30)=1.18, p=.246)$, nor for error trials at Time $1(t<1)$. It was only for error trials at Time 2 where the intervention groups showed a clear trend at Time $2(t(30)=1.974, p=.058)$, which apparently was due to an increase over time in the psychoeducation group from $.44 \mu \mathrm{V}$ to $1.05 \mu \mathrm{V}(t(15)=2.163, p=.047)$, while the mindfulness intervention group did not alter over time $(t(15)=1.265, p=$ $.225)$.

Just as the number of trials contributing to the ERN did not reliability relate to the variance in the ERN amplitude, number of trials did not systematically relate to the Pe amplitude, with $r$ 's of .259 and $-.198(p=.15$ and .28$)$ for the two sessions, respectively.

In terms of behavioral effects, for reaction time (RT) there was no significant effect of Group or any interactions with Group (all $F_{\mathrm{S}}<1$ ), nor any overall effect of Time $(F<1$ ). However, there was a clear Time $\times$ Intervention trend $(F(1$, $30)=3.91, p=.057$ ), whereby those in the mindfulness training group speeded up their responses from 535 to $514 \mathrm{~ms}$, 


\section{Mindfulness}

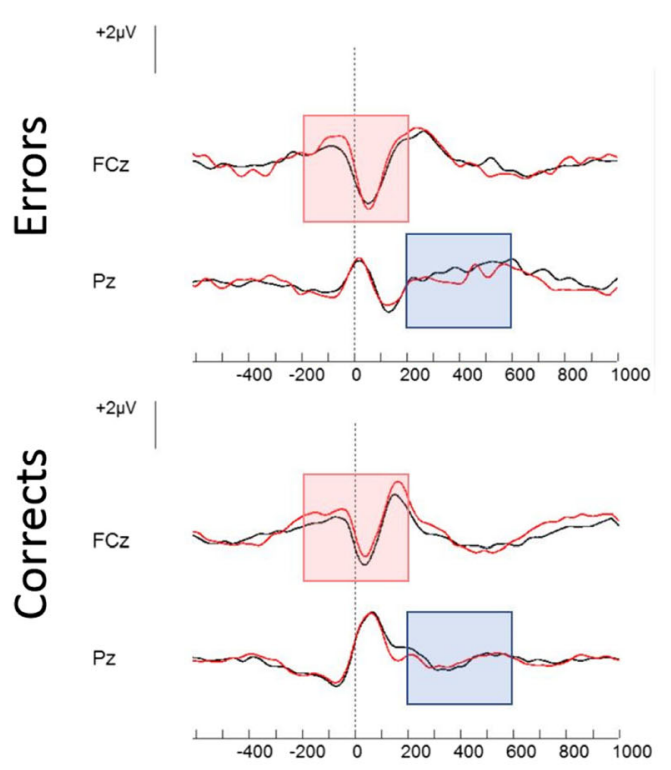

PsychoEducation

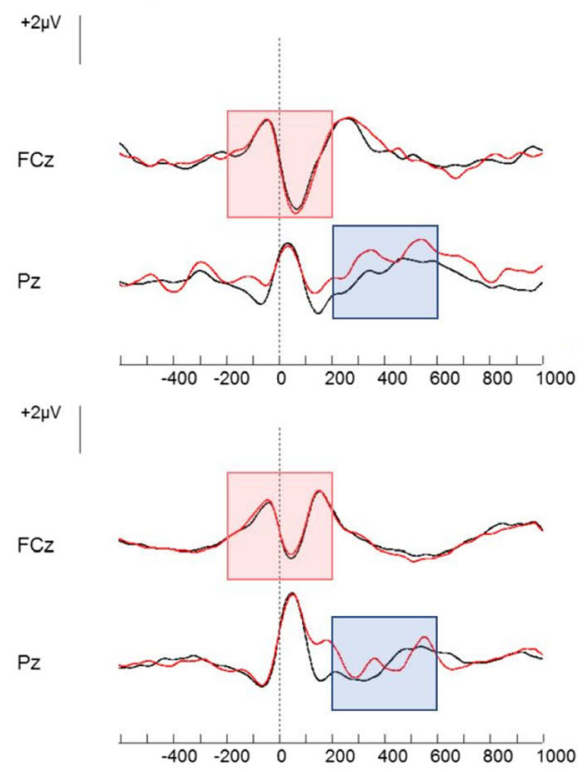

\section{Pre-Intervention}

Post-Intervention

Fig. 2 Waveforms time-locked to response time on the flanker task for waveforms on error and correct incongruent trials for the Mindfulness and Psychoeducation training groups pre- and post-treatment. The error- related negativity $(\mathrm{ERN})$ is the peak-to-peak difference measured at $\mathrm{FCz}$ within the red box and the error positivity (Pe) as the average amplitude in the 200- to $600-\mathrm{ms}$ period shown by the blue box, scored at $\mathrm{Pz}$ while those in the psychoeducation condition slowed down from 502 to $511 \mathrm{~ms}$. Despite the difference in RT at the preintervention time, the difference was not reliable $(F<1)$. Accuracy improved generally over time $(F(1,30)=19.1, p$ $<.001$, partial eta-squared $=.390)$, without regard to Intervention $(F<1)$, Group $(F(1,30)=2.31, p=.139$, partial eta-squared $=.072)$, or their interaction $(F<1)$ - in other words, a practice effect.

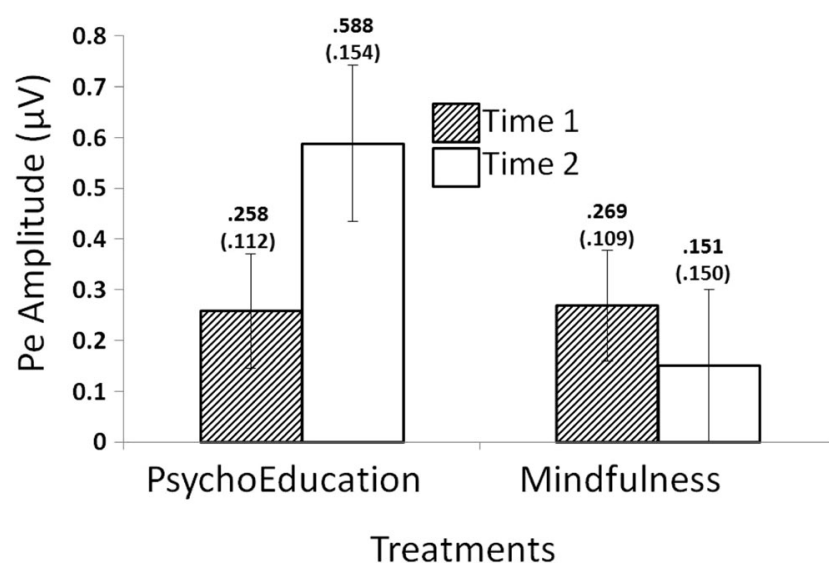

Fig. 3 Collapsing across diagnostic groups, a significant time-byintervention interaction on the error positivity (Pe), increasing after psychoeducation and decreasing after mindfulness training. Figures above the bars are mean values with standard errors in parentheses; the latter are also shown by vertical lines
Post-error slowing is seen as a marker of performance monitoring, although it may require a very large number of trials to be reliable. We found that post-error slowing was greater at Time 1 than Time 2 over all participants in that response times were $516 \mathrm{~ms}$ on trials following errors and $501 \mathrm{~ms}$ on trials following corrects at Time 1, while response times were $499 \mathrm{~ms}$ for trials following both corrects and errors at Time 2. This interaction was significant, $F(1,27)=5.94, p=.022$. However, there were no significant effects involving treatment type, group, or their interaction, $F$ 's $<1$.

\section{Self-report measures}

Means and standard deviations for each of the self-report measures are presented in Table 2. Each of the key variables anxiety, negative mood regulation, and mindfulness (specifically Non-Judge and Non-React) - were moderately to highly correlated at both time 1 and time 2. Given the relationships between variables, and the fact that the data included multiple time-points and multiple variables, we employed a doubly multivariate MANOVA approach, which consisted of an omnibus MANOVA followed by ANCOVAs to ascertain unique effects (Tabachnick \& Fidell, 2013). Averaging across timepoints, there were no significant omnibus multivariate effects for Group, Intervention, or their interaction (all $p>.05$ ). Looking at participants pre-/post-intervention, there was a 
Table 2. Descriptive statistics for self-report measures pre- and postintervention $(\mathrm{n}=36)$

\begin{tabular}{lllll}
\hline & $\begin{array}{l}\text { AMAS } \\
(M, S D)\end{array}$ & $\begin{array}{l}\text { NMR } \\
(M, S D)\end{array}$ & $\begin{array}{l}\text { N-J } \\
(M, S D)\end{array}$ & $\begin{array}{l}\text { N-R } \\
(M, S D)\end{array}$ \\
\hline Pre-test & $8.28(6.54)$ & $113.47(19.80)$ & $32.97(5.72)$ & $\begin{array}{l}24.25(4.29) \\
\text { Post-test }\end{array}$ \\
\hline
\end{tabular}

AMAS Adult Manifest Anxiety Scale-Elderly Version, Raw Scores (Lowe \& Reynolds, 2006), NMR Negative Mood Regulation Scale (Catanzaro \& Mearns, 1990), $N-J$ and $N-R$ Non-Judge and Non-React subscales from the Five Facet Mindfulness Questionnaire (Baer et al., 2006)

significant multivariate effect of time (Wilks' lambda $=.700$, $F(4,29)=3.11, p=.030$, partial eta squared $=.300)$, with a significant direct effect on the Adult Manifest Anxiety Scale $(F(1,29)=6.05, p=.020$, partial eta squared $=.173)$ and an indirect effect on the Non-Judge subscale of the Five Facet Mindfulness Questionnaire $(F(1,29)=7.31, p=.011$, partial eta squared $=.201)$. No main effects or interactions including intervention type were found.

\section{Discussion}

Despite the burgeoning literature demonstrating important neural and psychological sequelae of mindfulness training, there is an incomplete understanding of the mechanisms by which such effects occur. In the current study, we specifically focused on performance monitoring as a way of indexing the purported self-regulatory effects of mindfulness, by examining ERP components of performance monitoring as well as behavioral and self-report of psychological functioning. We did so in a sample of healthy older adults; normal aging shows declines in the performance monitoring system, and prevention-intervention with healthy seniors could allow them to capitalize on available cognitive reserve (Stern, 2009) to slow the rate of future decline. We had secondary hypotheses that pertained to a subgroup of our sample classified with subjective cognitive decline who exhibited significant concern or worry about perceived cognitive decline. We failed to find evidence in support of either significant performance monitoring differences in the subjective cognitive decline group at baseline, or selective intervention effects favoring this group. Therefore, the subsequent discussion of results focuses on our primary hypotheses collapsing across diagnostic groups and looking solely at intervention effects.

\section{Impact of mindfulness training on performance monitoring}

The current findings contribute to the accumulating evidence demonstrating that mindfulness training has an impact on performance monitoring (considered here as a fundamental aspect of self-regulation). Table 3 compares the results of the current study with those already discussed, in terms of changes in the ERN and Pe associated with mindfulness. As the table illustrates, rather than producing some absolute, consistent increase or decrease in error processing, the effects of mindfulness seem to vary as a function of length of exposure to practice as well as baseline cognitive function and error processing, and perhaps also participant group (e.g., different types of clinical populations).

Neural and behavioral effects In terms of neural effects, we found increased ERN amplitude but no reliable change in the Pe amplitude following mindfulness training. Larson et al. (2013) and Bing-Canar and others (2016) found that 15 min was too brief an exposure to increase the ERN, whereas Teper and Inzlicht (2013) found stable ERN increases in longer-term practitioners. These findings, taken together with those of the current study, might suggest that a longer exposure to mindfulness is required to show an impact on the ERN. That said, when taught to focus specifically on emotional stimuli, Saunders et al. (2016) found an increase in the ERN after an approximate 15-min intervention. This suggests that there is a particular relationship between reacting to emotional stimuli and generation of the ERN. As noted above, however, the functional significance of the ERN continues to be debated, so it is still difficult to make conclusive statements in this regard. It is interesting to note that, in the course of standard mindfulness-based stress reduction (and certainly for the current intervention), the first half of the course entails participants engaging in focused attention mindfulness, learning how to re-orient their attention to the breath and the present moment regardless of stimulus type. In the second half of the course, as participants become more familiar with the meditation techniques, they start to become more aware of how both selfreferential thoughts as well as feelings can trigger negative emotional reactions. This is the time when non-judgmental awareness is emphasized, and participants are often taught loving-kindness practice, as was the case for the current intervention. Considering the prior literature, this leads to the question, for there to be an impact on the ERN, is it the dose that is important, or the focus specifically on emotional stimuli? Perhaps one way to address this question in the future could be to have participants undergo the standard 8 weeks of mindfulness-based stress reduction, but then assess the ERN not only at pre-/post-intervention but also midway through the course, before loving-kindness and other emotion-specific practices have been introduced.

That said, it might still be challenging to dissociate whether particular stimuli impact the ERN in the context of mindfulness training. The Saunders et al. (2016) study specifically asked participants to only focus on cognitive versus emotional stimuli, and examined the ERN response. However, this does not necessarily translate to what happens when people practice mindfulness in a sustained way. In that context, individuals often find that thoughts in and of themselves - even when not emotionally laden - can nevertheless trigger an emotional reaction, simply because 
Table 3 Summary of current results in comparison to prior findings on performance monitoring associated with mindfulness training interventions of varying lengths

\begin{tabular}{|c|c|c|c|c|}
\hline & Length of Exposure & Population & $\mathrm{ERN}(+/-)$ & $\operatorname{Pe}(+/-)$ \\
\hline Current study: Time $\times$ Intervention & 8 weeks & $\begin{array}{l}\text { Older adults with and } \\
\text { without SCD }\end{array}$ & $\mathrm{MT}>\mathrm{PE}(+)$ & $\begin{array}{l}\text { MT (no change); } \\
\text { PE (+) }\end{array}$ \\
\hline Larson et al. (2013) & $15 \mathrm{~min}$ & Healthy volunteers & No group difference & MT > Control (-) \\
\hline Saunders et al. (2016) & $15 \mathrm{~min}$ & Healthy volunteers & $\mathrm{EF}(+) ; \mathrm{TF}$ (no change) & No group difference \\
\hline Bing-Canar et al. (2016) & $15 \mathrm{~min}$ & Healthy volunteers & No group difference & No group difference \\
\hline Schoenberg et al. (2014) & 12 weeks & Persons with ADHD & MT > Control (-) & $\begin{array}{l}\text { MT > } \\
\text { Control (+) }\end{array}$ \\
\hline Teper \& Inzlicht (2013) & Experienced $(M>3$ years $)$ & Healthy volunteers & MT > Controls $(+)$ & No group difference \\
\hline
\end{tabular}

$S C D$ subjective cognitive decline, $M T$ mindfulness training, $P E$ psychoeducation, $E F$ emotion-focused participants, $T F$ thought-focused participants, $(+)$ increasing amplitude, (-) decreasing amplitude, $(>)$ greater than

people become frustrated that they have engaged in mind-wandering. This is dissociable from the category of self-referential thoughts that lead directly to a negative emotion (e.g., depressive rumination). As such, it may prove more elusive trying to dissociate the impact of cold cognitive versus emotional stimuli within the experience of mindfulness practice beyond an experimental manipulation such as that of Saunders et al. (2016). In a way, this is not that surprising, given that the functional significance of the ERN itself (i.e., cognitive vs. affective response) continues to be hotly contested.

The current study does suggest that 8 weeks of consistent mindfulness training is a sufficient "dose" of practice to produce malleability in the performance monitoring system in healthy older adults. Furthermore, by interpreting the results from the two components, the findings suggest to us that mindfulness training led to an increase in attention related to errors without a concomitant increase in a negative affective response to those errors, although we accept that the functional significance of these components is still being debated. This was consistent with our hypotheses, and is consistent with what one would predict based on the notion that mindfulness training teaches one to "respond, not react." The notion that our findings support the ability to respond, not react is further corroborated by our finding of decreased anxiety post-intervention, suggesting that an increase in attention to errors did not come at a cost of increased negative affect (Aarts \& Pourtois, 2015; Hajcak, 2012; Olvet \& Hajcak, 2008).

Obtaining these results in an older adult population is particularly intriguing. The performance monitoring system declines with normal aging, yet current results demonstrate functional plasticity in this system is possible in the short duration of our intervention. While many other studies have shown functional (and structural) brain changes following 8 weeks of mindfulness training, these tend to be in younger or middle-aged adults. Research suggests that training-induced neuroplasticity may occur on longer time-scales in older adults (Jellinger \& Attems, 2013), so the fact that we were able to detect significant effects in 8 weeks is highly encouraging. Furthermore, developmental literature indicates that the regulation of cognition and emotion become competing demands in older adulthood, and older adults will often devote greater resources toward maintaining positive affect even when it comes at a cognitive cost (Peters et al., 2007), consistent with the so-called "positivity bias" (Mather \& Carstensen, 2005). However, the current findings suggest that mindfulness can improve both cognitive and emotional regulation simultaneously, which may be of particular utility to older adults. This is important given that comparatively little work has been done thus far to examine the impact of short-term mindfulness training in older adults (Gard, Hölzel, \& Lazar, 2014; Luders, 2014).

We previously reported that the P300 derived from the go trials in a go/no-go paradigm increased after mindfulness but not after psychoeducational training in this same group of participants (Smart et al., 2016), and yet we did not find the Pe, derived from the flanker task in the current study, to increase after mindfulness training. Given that the Pe could be considered to be a P300 response to the realization that one has made an error (Davies et al., 2001), one might have expected a similar finding for the two components. However, we caution against such a similarity being taken too literally. The P300 sensitivity to mindfulness training we reported earlier was only observed in the subjective cognitive decline group (and not the healthy controls), and was derived from trials time-locked to an external stimulus in an attention-intensive task. The Pe is elicited not to an external stimulus but to the internal realization that an error is about to be made. Of course, the amplitude of the Pe has also been linked to affective factors, as we have argued here, in a way that a series of go/nogo trials probably is not, and therefore the two components are not fully parallel. As such, the findings of our two studies taken together provide complementary evidence about the impact of mindfulness training on different cognitive processes, specifically, attention capacity and performance monitoring.

Self-report of psychological effects In terms of self-report measures, anxiety was the only construct for which we found 
direct effects post-intervention. Both groups showed a reduction in anxiety post-intervention, which is different from the specific main effect of mindfulness training on the aforementioned performance monitoring components. It is possible that self-reported anxiety reduction was merely due to repeat exposure to the laboratory environment and EEG/ERP data collection procedures, rather than an effect due to mechanisms of the interventions per se, which would explain why both groups decreased self-report of anxiety. It is also possible that receiving any form of intervention provided attention and care from a professional, which led to anxiety reduction across all participants. However, it is equally possible that both interventions significantly impacted anxiety, albeit via different mechanisms. For example, prior literature has already documented positive effects of various forms of mindfulness training on anxiety symptoms (Hoffman, Sawyer, Witt, \& Oh, 2010), while recent work indicates the decrease in negative affect associated with normalization of cognitive complaints following participation in the Memory and Aging program (our psychoeducation control condition) (Vandermorris et al., 2016). The important observation, then, is that there could be multiple interventions that could positively impact an individual's psychological state, but at least in the context of this study, only mindfulness training led to observable enhancements on objective measures of performance monitoring concurrent with those psychological changes.

Additionally, in terms of mindfulness effects, both interventions reduced non-judgment, but this was an indirect effect via anxiety. Non-Judge items on the Five Facet Mindfulness Questionnaire included statements such as "I believe some of my thoughts are abnormal or bad and I shouldn't think that way" and "I make judgments about whether my thoughts are good or bad." Both interventions provided discussion on cognitive aging and normalization of age-related cognitive slips, which likely decreased anxiety about cognitive performance. Non-judgment in this case, then, likely pertained to non-judgment of age-related cognitive abilities; from this point of view, it is straightforward to see why reduced anxiety would contribute to a decrease in nonjudgment specifically in older adults. That said, given that the anxiety reduction was non-specific to intervention type, the current results cannot be used to conclusively support the functional significance of changes in the ERN and Pe, which continues to be a subject of scientific debate.

We failed to detect significant changes in negative mood regulation following intervention. While mindfulness has been shown to enhance emotion regulation abilities, a majority of these studies are conducted on younger or middle-aged adults. Conversely, the developmental literature suggests that emotion regulation abilities actually improve with advanced age (e.g., Mather \& Carstensen, 2005; Carstensen et al., 2011), suggesting that the effects of mindfulness on older adults' emotion regulation may be subtler than other age groups or might require a longer retest window to show more dramatic effects.

\section{Limitations and future directions}

While the current results show short-term training effects after as little as 8 weeks of training, the long-term impact - and presumably preventative benefits - remains currently unknown. Future replications of this work should consider longer-term follow-up to address this issue. Moreover, while a rigorous randomized controlled trial design with repeated measures effects was employed in this study, the small sample size used indicates the need for future replications with larger samples to ascertain the reliability of the observed effects. Additionally, the current sample was comprised of a relatively homogeneous group of healthy (i.e., non-demented), well-educated, Caucasian individuals. While this homogeneity supported the internal validity of our design, questions remain regarding the generalizability to more diverse samples, which should again be addressed in future replications of this work.

\section{Conclusions}

The current results suggest that neural and behavioral plasticity in the performance monitoring system is evident after only 8 weeks of mindfulness training, and adds to the emerging body of literature illuminating the mechanisms by which mindfulness exerts its salutary effects on cognitive and emotional functioning. Moreover, observing these effects in older adults (including those who may be at risk for non-normal cognitive decline) supports the utility of pursuing nonpharmacologic interventions that could serve to provide a buffer against or attenuate the rate of any incipient cognitive decline. This has implications not only for older adults themselves, but also in terms of costs to the healthcare system.

Acknowledgements This study was supported by funding from the Alzheimer Society of Canada (Young Investigator Award \#1216 to CMS). The authors are grateful to the following for their help with data collection for this study: Katrina Blommaert, Coral Candlish-Rutherford, Esther Direnfeld, Andrew Fortune, Drew Halliday, Julie Irwin, Sasha Jouk, Janet Love, Jenny MacSween, Bryce Mulligan, Lara Oberg, Mark Rajandran, Dawn-Lee Ricard, Chris Prechotko, Stacey Ross, Thomas Ross, Jennifer Tippett, and Vanessa Vaartnou. We also thank James Desjardins, Meghan Weissflog, and Xin Zheng for their help with the processing of the data.

Parts of this manuscript were presented at the 41st Annual Meeting of the International Neuropsychological Society, Waikoloa, HI, USA (February, 2013) and the 25 Annual Neuroscience Conference of the Rotman Research Institute, Toronto, Ontario, Canada (March 2013).

\section{References}

Aarts, K., \& Pourtois, G. (2015). Error monitoring under negative affect: A window into maladaptive self-regulation processes. In G. H. E. Gendolla, M. Tops, \& S. L. Koole (Eds.), Handbook of 
Biobehavioral Approaches to Self-Regulation (pp. 109-124). New York: Springer.

Azulay, J., Smart, C. M., Mott, T., \& Cicerone, K. D. (2013). A pilot study examining the effect of mindfulness-based stress reduction on symptoms of chronic mild traumatic brain injury/post-concussive syndrome. The Journal of Head Trauma Rehabilitation, 28, 323-31.

Baer, R. A., Smith, G. T., Lykins, E., Button, D., Krietemeyer, J., Sauer, S., ... Williams, J. M. G. (2008). Construct validity of the Five Facet Mindfulness Questionnaire in meditating and non-meditating samples. Assessment, 15, 329-342.

Bing-Canar, H., Pizzuto, J., \& Compton, R. J. (2016). Mindfulness-ofbreathing exercise EEG alpha activity during cognitive performance. Psychophysiology, 53, 1366-1376.

Bishop, S. R., Lau, M., Shapiro, S., Carlson, L., Anderson, N. D., Carmody, J., Segal, Z. V., Abbey, S., Speca, M., Velting, D., \& Devins, G. (2004). Mindfulness: A Proposed operational definition. Clinical Psychology: Science and Practice, 11(3), 230-241. doi: https://doi.org/10.1093/clipsy/bph077

Bohlmeijer, E., Prenger, R., Taal, E., \& Cuijpers, P. (2010). The effects of mindfulness-based stress reduction therapy on mental health of adults with a chronic medical disease: A meta-analysis. Journal of Psychosomatic Research, 68, 539-544.

Carp, J., \& Compton, R. J. (2009). Alpha power is influenced by performance errors. Psychophysiology, 46, 336-343. doi: https://doi.org/ 10.1111/j.1469-8986.2008.00773.x

Carstensen, L. L., Turan, B., Scheibe, S., Ram, N., Ersner-Hershfield, H., Samanez-Larkin, G. R., Brooks, K. P., \& Nesselroade, J. R. (2011). Emotional experience improves with age: Evidence based on over 10 years of experience sampling. Psychology and Aging, 26, 21-33.

Catanzaro, S. J., \& Mearns, J. (1990). Measuring generalized expectancies for negative mood regulation: Initial scale development and implications. Journal of Personality Assessment, 54, 546-563.

Chen, A. J-W., Novakovic-Agopian, T., Nycum, T. J., Song, S., Turner, G. R., Hills, N. K., Rome, S., Abrams, G. M., \& D'Esposito, M. (2011). Training of goal-directed attention regulation enhances control over neural processing for individuals with brain injury. Brain

Chiesa, A., Calati, R., \& Serretti, A. (2011). Does mindfulness improve cognitive abilities? A systematic review of neuropsychological findings. Clinical Psychology Review, 31, 449-464.

Chiesa, A., Serretti, A., \& Jakobsen, J. C. (2013). Mindfulness: top-down or bottom-up emotion regulation strategy? Clinical Psychology Review, 33, 82-96.

Davies, P. L., Segalowitz, S. J., Dywan, J., \& Pailing, P. E. (2001). Errorrelated negativity and positivity as they relate to other ERP indices of attentional control and stimulus processing. Biological Psychology, 56, 191-206.

Desjardins, J. A., \& Segalowitz, S. J. (2013). The timing and consistency of event-related potential responses when processing faces: Deconstructing the P100-N170 complex using ICA and robust estimation. Journal of Vision, 13, 1-18.

Diekhof, E. K., Falkai, P., \& Gruber, O. (2011). The orbitofrontal cortex and its role in the assignment of behavioural significance. Neuropsychologia, 49, 984-91.

Eriksen, B.A., \& Eriksen, C.W. (1974). Effects of noise letters in the identification of target letters in a nonsearch task. Perception and Psychophysics, 16, 143- 149.

Etkin, A., Egner, T., \& Kalisch, R. (2011). Emotional processing in anterior cingulate and medial prefrontal cortex. Trends in Cognitive Sciences, 15, 85-93.

Farb, N. A., Segal, Z. V., Mayberg, H., Bean, J., McKeon, D., Fatima, Z., \& Anderson, A. K. (2007). Attending to the present: Mindfulness meditation reveals distinct neural modes of self-reference. Social Cognitive Affective Neuroscience, 2, 313-322.

Friedman, D. (2012). The components of aging. In S. J. Luck \& E. S. Kappenman (Eds.), Oxford Handbook of Event-Related Potentials (513-536). New York: Oxford University Press.
Gard, T., Hölzel, B. K., \& Lazar, S. W. (2014). The potential effects of meditation on age-related cognitive decline: a systematic review. Annals of the NY Academy of Sciences, 1307, 89-103.

Gehring, W. J., Liu, Y., Orr, J. M., \& Carp, J. (2012). The error-related negativity (ERN/Ne). In S. J. Luck \& E. S. Kappenman (Eds.), The Oxford Handbook of Event-Related Potential Components (pp. 231-291). New York: Oxford.

Hajcak, G. (2012). What we've learned from mistakes: Insights from error-related brain activity. Current Directions in Psychological Science, 21, 101-106.

Hofmann, S.G., Sawyer, A.T., Witt, A.A. \& Oh, D. (2010). The effect of mindfulness-based therapy on anxiety and depression: A metaanalytic review. J Consult Clin Psychol, 78(2), 169-183. doi: https://doi.org/10.1037/a0018555

Holroyd, C. B., \& Yeung, N. (2012). Motivation of extended behaviors by anterior cingulate cortex. Trends in Cognitive Sciences, 16, 122-8.

Jellinger, K. A., \& Attems, J. (2013). Neuropathological approaches to cerebral aging and neuroplasticity. Dialogues in Clinical Neuroscience, 15, 29-43.

Jessen, F., Amariglio, R. E., van Boxtel, M., Breteler, M., Ceccaldi, M., Chételat, G., ... Subjective Cognitive Decline Initiative (SCD-I) Working Group (2014). A conceptual framework for research on subjective cognitive decline in preclinical Alzheimer's disease. Alzheimer's \& Dementia. doi: https://doi.org/10.1016/j.jalz.2014. 01.001

Kabat-Zinn, J. (1990). Full catastrophe living: Using the wisdom of your body and mind to face stress, pain, and illness. New York: Delta.

Kit, K. A., Tuokko, H. A., \& Mateer, C. A. (2008). A review of the stereotype threat literature and its application in a neurological population. Neuropsychology Review, 18, 132-148.

Larson, M. J., Steffens, P. R., \& Primosch, M. (2013). The impact of a brief mindfulness meditation intervention on cognitive control and error-related performance monitoring. Frontiers in Human Neuroscience, 9:7. doi:https://doi.org/10.3389/fnhum.2013.00308

Levine, B., Schweizer, T. A., O'Connor, C., Turner, G. R., Gillingham, S., Stuss, D. T., Manly, T., \& Robertson, I. H. (2011). Rehabilitation of executive functioning in patients with frontal lobe brain damage with Goal Management Training. Frontiers in Human Neuroscience, 5:9. doi:https://doi.org/10.3389/fnhum.2011.00009

Lowe, P. A., \& Reynolds, C. R. (2006). Examination of the psychometric properties of the Adult Manifest Anxiety Scale-Elderly Version scores. Educational and Psychological Measurement, 66, 93-115.

Luders, E. (2014). Exploring age-related brain degeneration in meditation practitioners. Annals of the NY Academy of Sciences, 1307, 82-88.

Lutz, A., Slagter, H. A., Dunne, J. D., \& Davidson, R. J. (2008). Attention regulation and monitoring in meditation. Trends in Cognitive Sciences, 12, 163-169.

MacLean, K. A., Ferrer, E., Aichele, S. R., Bridwell, D. A., Zanesco, A. P., Jacobs, T. L., ... Saron, C. D. (2010). Intensive meditation training improves perceptual discrimination and sustained attention. Psychological Science, 21, 829-839.

Mather, M., \& Carstensen, L. L. (2005). Aging and motivated cognition: The positivity effect in attention and memory. Trends in Cognitive Sciences, 9, 496-502.

Nieuwenhuis, S., Ridderinkhof, K. R., Talsma, D., Coles, M. G. H., Holroyd, C. B., Kok, A., \& van der Molen, M. W. (2002). A computational account of altered error processing in older age: Dopamine and the error-related negativity. Cognitive, Affective, \& Behavioral Neuroscience, 2, 19-36.

Novakovic-Agopian, T., Chen, A. J-W., Rome, S., Abrams, G., Castelli, H., Rossi, A., McKim, R., Hills, N., \& D’Esposito, M. (2010). Rehabilitation of executive functioning with training in attention regulation applied to individually defined goals: A pilot study bridging theory, assessment, and treatment. Journal of Head Trauma Rehabilitation, 26, 325-338. 
Olvet, D. M., \& Hajcak, G. (2008). The error-related negativity (ERN) and psychopathology: Toward an endotype. Clinical Psychology Review, 28, 1343-1354.

Overbeek, T. J. M., Nieuwenhuis, S., \& Ridderinkhof, K. R. (2005). Dissociable components of error processing: On the functional significance of the Pe vis-à-vis the ERN/Ne. Journal of Psychophysiology, 19, 319-329.

Peters, E., Hess, T. M., Västfjäll, D., \& Auman, C. (2007). Adult age differences in dual information processes: Implications for the role of affective and deliberative processes in older adults' decision making. Perspectives on Psychological Science, 2, 1-23.

Pietschmann, M., Simon, K., Endrass, T., \& Kathmann, N. (2008). Changes of performance monitoring with learning in older and younger adults. Psychophysiology, 45, 559-568.

Polli, F. E., Barton, J. J. S., Cain, M. S., Thakkar, K. N., Rauch, S. L., \& Manoach D. S. (2005). Rostral and dorsal anterior cingulate cortex make dissociable contributions during antisaccade error commission. Proceedings of the National Academy of Sciences U.S.A., 102, 15700-15705.

Psychology Software Tools (2012). E-Prime 2.0 Sharpsburg: Psychology Software Tools.

Reisberg, B., Prichep, L., Mosconi, L., John, E. R., Glodzik-Sobanska, L., Boksay, I., ... de Leon, M. J. (2008). The pre-mild cognitive impairment, subjective cognitive impairment stage of Alzheimer's disease. Alzheimer's \& Dementia, 4, S98-108.

Ridderinkhof, K. R., Ullsperger, M., Crone, E. A., \& Nieuwenhuis, S. (2004). The role of the medial frontal cortex in cognitive control. Science, 306, 443-447.

Ridderinkhof, K. R., van den Wildenberg, W. P., Segalowitz, S. J., \& Carter, C. S. (2004). Neurocognitive mechanisms of cognitive control: the role of prefrontal cortex in action selection, response inhibition, performance monitoring, and reward-based learning. Brain \& Cognition, 56, 129-40.

Santesso, D. L., \& Segalowitz, S. J. (2009). The error-related negativity is related to risk taking and empathy in young men. Psychophysiology, 46, 143-152.

Saunders, B., Rodrigo, A. H., \& Inzlicht, M. (2016). Mindful awareness of feelings increases neural performance monitoring. Cognitive, Affective, \& Behavioral Neuroscience, 16, 93-105.

Schnider, A, Treyer, V, \& Buck, A. (2005). The human orbitofrontal cortex monitors outcomes even when no reward is at stake. Neuropsychologia, 43, 316-23.

Schoenberg, P. L. A., Hepark, S., Kan, C. C., Barendregt, H. P., Buitelaar, J. K., \& Speckens, A. E. M. (2014). Effects of mindfulness-based cognitive therapy on neurophysiological correlates of performance monitoring in adult attention-deficit hyperactivity disorder. Clinical Neurophysiology, 125, 1407-1416.

Segal, Z., Williams, M., \& Teasdale, J. (2012). Mindfulness-based cognitive therapy for depression (2nd). New York: Guilford.

Segalowitz, S. J., Santesso, D. L., Willoughby, T., Reker, D. L., Campbell, K., Chalmers, H., \& Rose-Krasnor, L. (2012). Adolescent peer interaction and trait surgency weaken medial prefrontal cortex responses to failure. Social Cognitive and Affective Neuroscience, 7, 115-124.

Smart, C. M. (2012). Wisdom Mind: Mindfulness training as the path to basic sanity. University of Victoria, Victoria, BC: Unpublished clinical manual.
Smart, C. M., Segalowitz, S. J., Mulligan, B. P., Koudys, J., \& Gawryluk, J. (2016). Mindfulness training for older adults with subjective cognitive decline: Results from a pilot randomized controlled trial. Journal of Alzheimer's Disease, 52, 757-774.

Smart, C. M., Segalowitz, S. J., Mulligan, B. P., \& MacDonald, S. W. S. (2014). Attention capacity and self-report of subjective cognitive decline: A P300 ERP study. Biological Psychology, 103, 144-151.

Stern, Y. (2009). Cognitive reserve. Neuropsychologia, 47, 2015-2028.

Tabachnick, B. G., \& Fidell, L. S. (2013). Using multivariate statistics (6th). Harlow: Pearson Education.

Teper, R., \& Inzlicht, M. (2013). Meditation, mindfulness, and executive control: The importance of emotional acceptance and brain-based performance monitoring. Social Cognitive Affective Neuroscience, $8,85-92$.

Troyer, A. (2001). Improving memory knowledge, satisfaction, and functioning via an education and intervention program for older adults. Aging, Neuropsychology, and Cognition, 8, 256-268.

van Noordt, S. J., Campopiano, A., \& Segalowitz, S. J. (2016). A functional classification of medial frontal negativity ERPs: Theta oscillations and single subject effects. Psychophysiology, 53(9), 1317-1334.

van Noordt, S. J., Desjardins, J. A., Gogo, C. E., Tekok-Kilic, A., \& Segalowitz, S. J. (2017). Cognitive control in the eye of the beholder: Electrocortical theta and alpha modulation during response preparation in a cued saccade task. Neuroimage, 145(Pt A), 82-95.

van Noordt, S. J. R., Desjardins, J. A., \& Segalowitz, S. J. (2015). Watch out! Medial frontal cortex is activated by cues signalling potential changes in response demands. NeuroImage, 114, 356-370.

van Noordt, S. J. R., \& Segalowitz, S. J. (2012). Performance monitoring and the medial prefrontal cortex: a review of individual differences and context effects as a window on self-regulation. Frontiers in Neuroscience, 6, article 197.

van Veen, V., \& Carter, C. S. (2002). The anterior cingulate as a conflict monitor: fMRI and ERP studies. Physiology \& Behavior, 77, $477-$ 482.

Vandermorris, S., Davidson, S., Au, A., Sue, J., Fallah, S., \& Troyer, A. K. (2016). 'Accepting where I'm at' - a qualitative study of the mechanisms, benefits, and impact of a behavioral memory intervention for community-dwelling older adults. Aging \& Mental Health, doi: https://doi.org/10.1080/13607863.2016.1181709

Wechsler, D. (2008). Wechsler Adult Intelligence Scale, Fourth Edition. San Antonio, TX: Pearson Assessments.

Wiegand, M. A., Troyer, A. K., Gojmerac, C., \& Murphy, K. J. (2013). Facilitating change in health-related behaviours and intentions: A randomized controlled trial of a multidimensional memory program for older adults. Aging \& Mental Health, 17, 806-815.

Wild-Wall, N., Willemssen, R., \& Falkenstein, M. (2009). Feedbackrelated processes during a time-production task in young and older adults. Clinical Neurophysiology, 120, 407-413.

Zylowska, L., Ackerman, D.L., Yang, M.H., Futrell, J.L, Horton, N.L., Hale, T.S., Pataki, C. \& Smalley, S.L. (2008). Mindfulness meditation training in adults and adolescents with ADHD: A feasibility study. Journal of Attention Disorders, 11(6), 737-746. doi: https:// doi.org/10.1177/1087054707308502 